\title{
Location-based Mobile Learning Applications: A conceptual framework for co-design
}

\author{
Alejandra B. Lliteras ${ }^{1,2}$, Cecilia Challiol ${ }^{1,3}$, Silvia E. Gordillo ${ }^{1,2}$ \\ ${ }^{1}$ UNLP, Facultad de Informática, LIFIA \\ ${ }^{2} \mathrm{CICPBA}$ \\ La Plata, Buenos Aires, Argentina \\ Buenos Aires, Argentina \\ ${ }^{3}$ CONICET \\ Argentina \\ e-mails: \{lliteras, ceciliac, gordillo\}@lifia.info.unlp.edu.ar
}

\begin{abstract}
This paper presents a conceptual framework for codesign (by multidisciplinary team) Location-based Mobile Learning Applications. Four stages are identified related with this kind of applications: conceptual design, development cycle, put into practice and evaluation. This paper is focus on the first stage, the conceptual design. For this stage, relevant features are described. In particular, good practices of software engineering could be used for the design stage. As part of conceptual design, guidelines are provided to define learning content decoupled of relevant locations of physical space (indoor or outdoor). This allows to reuse both in different applications.
\end{abstract}

Index Terms-Location-based Mobile Learning Applications, Mobile Learning, Technology Enhanced Learning, In-situ Design, Separation of Concerns, Conceptual Framework, CoDesign, Co-Creation.

\section{INTRODUCCIÓN}

Actualmente el uso de los dispositivos móviles, como por ejemplo de smartphones o tablets, se ha convertido en algo habitual para las personas, independientemente de su edad. Éste ya no solo se limita a llamadas telefónicas o mensajes de texto, sino que además se usa para aplicaciones destinadas a diversos usos, por ejemplo: juegos, entretenimiento y educación [1].

Los avances tecnológicos en el área móvil (incluyendo a los dispositivos móviles) han revolucionado el panorama de enseñanza y aprendizaje [2]. Esto ha generado un cambio en los roles tanto de los alumnos como de los docentes, y adicionalmente abrió la posibilidad de su uso dentro y fuera del aula. Sin embargo, en [3] se menciona que aún existen falencias al momento de implementar, en educación, soluciones empleando tecnología móvil. En particular, muchas de las soluciones no involucran a los docentes en su diseño. La participación de los docentes en el diseño, les genera un nuevo desafío a los mismos [4]. La importancia de esta participación en el proceso de diseño ha sido explorada, por ejemplo, en [3], [4] y [5].

Al aprendizaje enriquecido con tecnología móvil se lo denomina Aprendizaje Móvil. Este concepto ha sido explorado por diferentes autores, por ejemplo, [6], [7], [8], [9], [10] y [11]. Estos autores coinciden en que este tipo de aprendizaje involucra el uso de tecnología móvil, más específicamente el uso de dispositivos móviles, en los cuales los alumnos reciben algún contenido educativo. Sin embargo, aún no se cuenta con una definición unificada para el concepto Aprendizaje Móvil. Cuando en este aprendizaje, la posición de los alumnos es un aspecto relevante, se lo denomina Aprendizaje Móvil basado en Posicionamiento [2]. En este tipo de aprendizaje es fundamental la participación de los docentes en el proceso de diseño in-situ [12], sin embargo lograr que los mismos trabajen de manera conjunta con investigadores y desarrolladores de software todavía sigue siendo un campo abierto de investigación. Más aún, en [13] y en [14] se menciona la necesidad de realizar programas de entrenamiento en Aprendizaje Móvil para que los docentes puedan adoptar este tipo de aprendizaje.

En los últimos años se han aplicado diferentes estrategias para abordar el diseño en el área de aprendizaje enriquecido con tecnología. Una propuesta metodológica para considerar diferentes aspectos relacionados a este diseño es presentado en [3], donde se identifican los siguientes aspectos: claridad, valor agregado, compatibilidad y tolerancia. Otros enfoques, incorporan el uso de diferentes conceptos de la ingeniería de software para su diseño. En [15] se promueve el uso de la separación de concerns, puntualmente para e-learning. En particular, para el área de aplicaciones de aprendizaje móvil contextual (tanto físico como social), en [2] se presenta un enfoque de diseño basado en tres componentes: facilitación, actividad in-situ y tecnología. En estos enfoques mencionados, se tienen acoplados los aspectos de posicionamiento y contenido educativo, lo que imposibilita el reuso de los mismos. Esta es la motivación principal para proponer el framework conceptual que se presentará en este trabajo.

El objetivo del trabajo es presentar un framework conceptual para el co-diseño de Aplicaciones Educativas Móviles basadas en Posicionamiento. Dicho framework brinda los lineamientos para diseñar el contenido educativo desacoplado de los lugares relevantes identificados dentro de un espacio físico (tanto indoor como outdoor). Esto facilita el reuso de ambos conceptos en diferentes aplicaciones. El framework brinda soporte para que un equipo multidisciplinario (por ejemplo, docentes, investigadores y desarrolladores) pueda participar en el diseño in-situ de este tipo de aplicaciones. Dicho framework está planteado usando 
el concepto de separación de concerns [16]. En este trabajo se describirán cada uno de los concerns (o capas) del framework, los cuales permiten ir construyendo el diseño conceptual, para luego plasmarlo en una aplicación móvil específica. Para estos concerns, se brindarán buenas prácticas de la ingeniería de software para facilitar así el reuso de todos los aspectos involucrados.

Además, se describirán las cuatro etapas identificadas en relación a las Aplicaciones Educativas Móviles basadas en Posicionamiento. En particular, el framework conceptual propuesto está focalizado en abordar la primera etapa, es decir, el diseño conceptual de este tipo de aplicaciones.

También se espera poder generar un espacio de discusión y reflexión acerca del uso de buenas prácticas para el diseño de este tipo de aplicaciones, como así también ayudar a la comprensión de los aspectos involucrados en las Aplicaciones Educativas Móviles basadas en Posicionamiento.

El trabajo se estructura de la siguiente manera. En la Sección 2 se presenta un ejemplo motivacional para mostrar los aspectos relacionados al diseño de Aplicaciones Educativas Móviles basadas en Posicionamiento. En la Sección 3 se presenta el framework conceptual propuesto. En la Sección 4 se discuten algunas características relacionadas al diseño de este tipo de aplicaciones como así también algunos trabajos relacionados. Las conclusiones y trabajos futuros se detallan en la Sección 5.

\section{MOTIVACIÓN}

En esta sección se presentan algunos aspectos relacionados a las Aplicaciones Educativas Móviles basadas en Posicionamiento. Estos aspectos deberían ser considerados al momento de diseñar este tipo de aplicaciones ya que pueden impactar no solo en el reuso de las mismas sino también en la manera en la que éstas evolucionan [17].

Supongamos que se cuenta con un equipo multidisciplinario formado por al menos expertos en educación y por expertos en tecnología, el cual desea co-diseñar una Aplicación Educativa Móvil basada en Posicionamiento, para ser usada dentro del "Jardín Botánico" de la ciudad de Buenos Aires [18]. En dicho lugar, se quiere brindar contenido educativo, en forma de preguntas de opción múltiple enfocadas al área de ciencias naturales. Estas preguntas estarán destinadas a alumnos de cuarto grado. Se desea que la aplicación brinde estas preguntas en determinados lugares relevantes (ya sean posiciones puntuales o áreas de interés) del espacio físico (en este caso el Jardín Botánico).

Para ser más precisos en el diseño de la aplicación, los miembros del equipo visitan el Jardín Botánico, para observar y determinar los lugares relevantes como también para analizar la posibilidad de movilidad dentro del mismo (esto permitirá luego proponerle al alumno ir de un lugar a otro a través de la Aplicación Educativa Móvil). A partir de esta observación el equipo podría generar una descripción gráfica del lugar (como se propone en [19]), por ejemplo, mediante un bosquejo o plano. En dicho bosquejo, se podrían especificar posibles elementos del lugar que se consideren relevantes (al estilo de lo presentado en [20], donde se los denomina landmarks). Por ejemplo, se podría indicar en el bosquejo donde está posicionado el cartel de bienvenida.

Mientras los miembros del equipo realizan el recorrido, deberán decidir cuáles son los lugares relevantes en los que posteriormente se brindarán las preguntas (mediante la Aplicación Educativa Móvil). Estas preguntas podrían estar planteadas usando conceptos generales o especificadas en base al entorno en el que luego serán brindadas (por ejemplo, requerirán que los alumnos observen alguna característica particular de alguna planta del lugar). Supongamos que el equipo multidisciplinario decide co-diseñar cuatro preguntas generales de opción múltiple.

Luego, el equipo deberá definir de que manera estructurar las preguntas creadas (diferentes tipos de estructuras son mencionadas en [21]). Por ejemplo, si se sigue una estructura lineal (es decir, luego de responder una pregunta se brinda la siguiente) o una estructura libre (los alumnos elijen en qué orden recibir las preguntas). Supongamos que el equipo elije estructurar, de manera lineal, tres de las cuatro preguntas creadas anteriormente. Además, el equipo define en que lugares relevantes se va a brindar cada elemento de la estructura definida.

Supongamos que el equipo además quiere crear una segunda Aplicación Educativa Móvil, que reuse el bosquejo creado, los lugares relevantes identificados y dos de las preguntas planteadas para la aplicación anterior, pero que le agregue la pregunta que aún no se había usado de las cuatro definidas inicialmente. Para esta segunda aplicación el equipo elije una estructura libre y define que elemento de la estructura definida se brindará en cada uno de lugares relevantes.

En la Fig. 1 se pueden observar los contenidos educativos mencionados (las cuatro preguntas), a su vez se muestran las dos estructuras señaladas (lineal y libre), donde cada elemento de la estructura indica a que pregunta hace referencia. Por otro lado, se puede ver el bosquejo del Jardín Botánico y los lugares relevantes identificados dentro del mismo. Además, se puede apreciar cómo se relacionan cada uno de los elementos de las estructuras con los lugares relevantes.

Se puede apreciar también en la Fig. 1 como diferentes aspectos se pueden reusar para generar así diferentes Aplicaciones Educativas Móviles. A partir de esto se identificó que:

- Las mismas preguntas (generales) se podrían reusar en diferentes estructuras

- Los elementos de una estructura se podrían posicionar en diferentes lugares relevantes

- En los mismos lugares relevantes se podrían brindar diferentes elementos de estructuras

El reuso de estos aspectos será posible siempre y cuando se considere el desacople de los mismos, tanto en la etapa de diseño como de implementación. A partir de esto, en este trabajo se define un Framework Conceptual para el co-diseño usando el concepto de separación de concerns para fomentar el reuso de los diferentes aspectos. Dicho framework se presentará en la siguiente sección. 


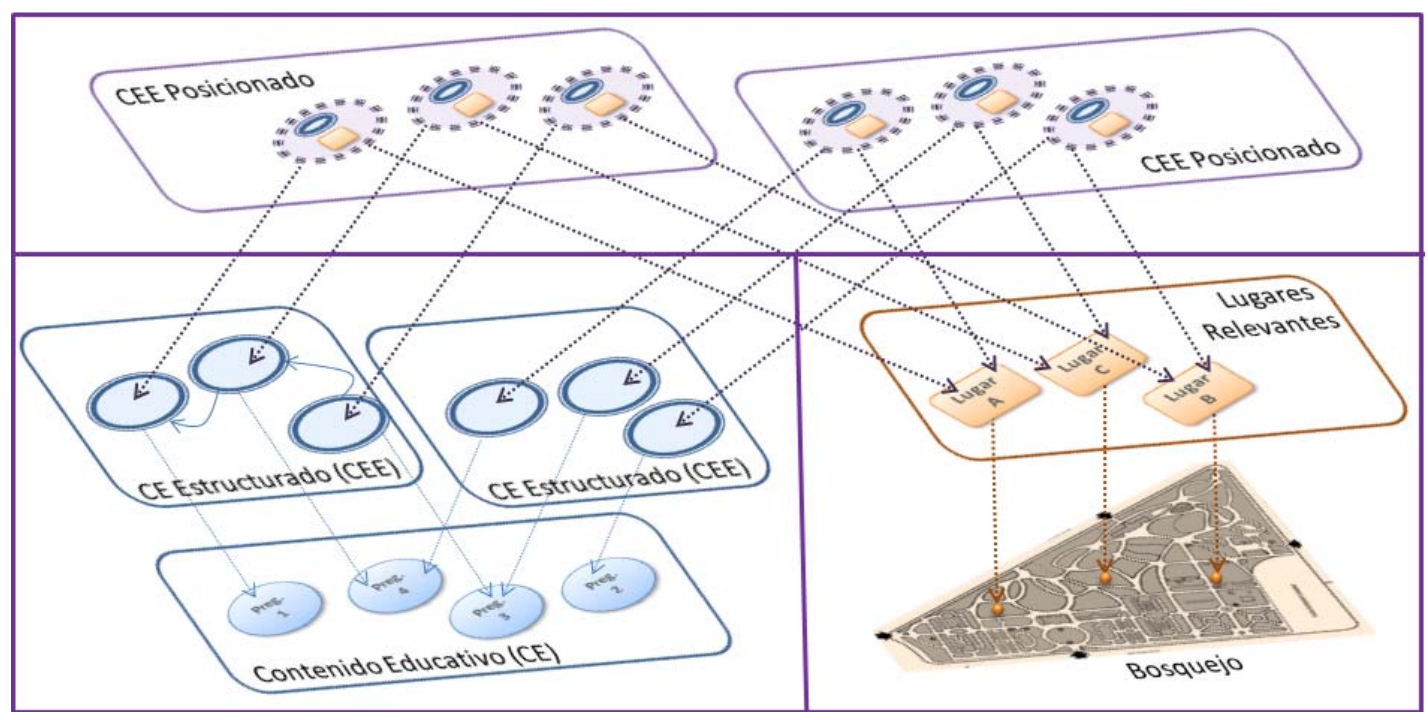

Fig. 1. Ejemplos de diseño en el "Jardín Botánico".

\section{FRAMEWORK CONCEPTUAL PROPUESTO}

En esta sección se presenta el Framework Conceptual propuesto para el co-diseño de Aplicaciones Educativas Móviles basadas en Posicionamiento. Este framework surge a partir de explorar diferentes aspectos relacionados a este tipo de aplicaciones. En [22] se presenta un enfoque de modelado para la creación de Aplicaciones Educativas Móviles basadas en Posicionamiento. En particular, este modelo explora como incluir elementos concretos en este tipo de aplicaciones. Cabe destacar que este modelo se basa en el concepto de separación de concerns para representar los aspectos de actividades educativas desacopladas del posicionamiento. Este enfoque está orientado a usuarios expertos en tecnología. En [22] se presenta, en base al enfoque de modelado, un prototipo de Aplicación Educativa Móvil basada en Posicionamiento que usa elementos concretos. En particular se detallan dos experiencias de puesta en práctica del mismo. Por otro lado, en [23] se presenta una herramienta de autor para crear Aplicaciones Educativas Móviles basadas en Posicionamiento, la cual es una extensión de la herramienta presentada en [24]. Esta herramienta usa códigos QR [25] y GPS [26] para posicionar el contenido educativo. Además la herramienta permite generar archivos XML [27] diferentes para cada aspecto relacionado a este tipo de aplicaciones (contenidos educativos, estructuras, posicionamiento y las relaciones entre los anteriores). Es decir, se explora la separación de concerns en este caso desde una herramienta de autor, fomentando el reuso de cada uno de los aspectos generados.

A partir de la exploración realizada en trabajos anteriores (por ejemplo, [22], [24] y [23]) y teniendo en cuenta la bibliografía existente sobre la temática, se identificaron cuatro etapas bien diferenciadas relacionadas a las Aplicaciones Educativas Móviles basadas en Posicionamiento:

- Diseño Conceptual de las mismas
- Ciclo de desarrollo usando tecnología móvil y contemplando el diseño conceptual creado

- Puesta en Práctica del desarrollo realizado

- Evaluación de la Puesta en Práctica

Cada una de estas etapas implica diferentes desafíos y requiere lineamientos particulares para llevarlas a cabo. En este trabajo se hará hincapié en el diseño conceptual de este tipo de aplicaciones, permitiendo así mostrar la primera versión del framework propuesto.

Cabe mencionar que de ahora en más se usará la terminología diseño y co-diseño de manera indistinta, entendiendo que cuando se habla de diseño, éste involucra a un equipo multidisciplinario.

En la Fig. 2 se puede apreciar el Framework Conceptual propuesto para abordar los diferentes concerns identificados en la etapa de diseño conceptual de Aplicaciones Educativas Móviles basadas en Posicionamiento.

A continuación se brinda más nivel de detalle de cada uno de los concerns destacados en la Fig. 2. En cada uno de los mismos, como se mencionó anteriormente, se debe considerar la participación de un equipo multidisciplinario.

\section{A. Diseño In-Situ del espacio físico}

En este tipo de diseño es necesario interactuar con el espacio físico real para poder generar un bosquejo del mismo, como así también identificar los lugares relevantes (donde posteriormente se podría brindar algún contenido educativo a los alumnos).

Cabe mencionar que un bosquejo constituye una porción de la realidad acorde a como es percibida por quien lo realiza [28]. $\mathrm{Al}$ ser este realizado por un equipo multidisciplinario se puede enriquecer desde la perspectiva de todos los integrantes del equipo. Es decir, este bosquejo es realizado mediante un codiseño (o lo que es lo mismo, es co-creado). Una forma de realizar un bosquejo, puede ser mediante una representación 


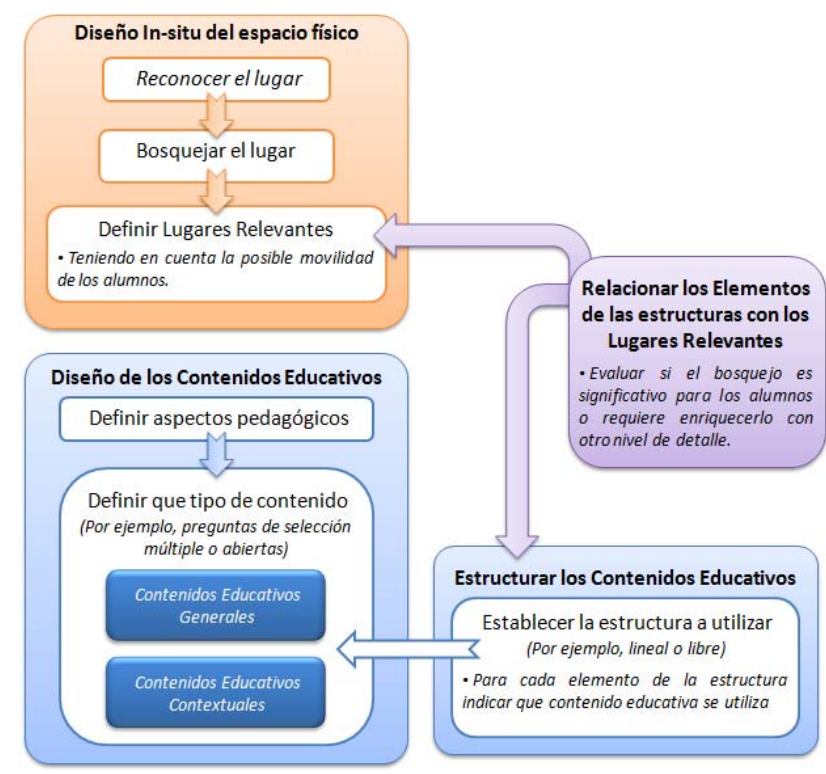

Fig. 2. Framework propuesto enfocado en la etapa de diseño conceptual.

gráfica [19]. En este tipo de representación, el foco esta puesto en los objetos y las relaciones entre ellos, sin entrar en detalles técnicos [29].

Los objetos incluidos en los bosquejos, son denominados generalmente "landmarks", y pueden servir como puntos de referencia [20]. Se debe tener en cuenta que estos landmarks podrían ser temporales, es decir, variar en el tiempo. Por ejemplo, una planta podría estar florecida o no dependiendo de la estación del año. Si esto es usado como un landmark se debe tener en cuenta esta variabilidad.

En el caso que este bosquejo este orientado a un grupo de alumnos en particular, esto podría afectar el reuso del mismo, ya que por ejemplo, podría no ser significativo para otro grupo de alumnos.

En relación a los lugares relevantes, es importante considerar que mecanismos de posicionamiento se pueden usar luego para detectar que el alumno está posicionado en alguno de ellos. Por ejemplo, si en el lugar funciona adecuadamente el GPS o si es viable tener códigos QR. Y además, estos lugares relevantes deben estar definidos considerando que puedan ser accesibles para los alumnos posteriormente (cuando estén usando la aplicación).

Las posiciones deben estar diseñadas independiente de los posibles contenidos educativos que luego se brinden ahí o de los mecanismos de posicionamiento usados. Esto brinda flexibilidad, para poder hacer el "weaving"1 (relación) entre los distintos concerns.

Los mecanismos de sensado son un punto de variabilidad, ya que estos pueden ir cambiando en el tiempo, esto debe ser tenido en cuenta a la hora de diseñar sobre todo por los expertos en tecnología. En [30] se aborda este tema, desde la

\footnotetext{
1 Concepto usado en la separación de concerns para relacionar elementos de distintos concerns para tener una aplicación funcionando.
}

perspectiva del diseño orientado a objetos y basándose en buenas prácticas de la ingeniería de software.

\section{B. Diseño de los Contenidos Educativos}

Los contenidos para este tipo de aplicaciones pueden ser diseñados desde cualquier lugar (por ejemplo, una oficina), o bien, pueden diseñarse in-situ [31]. El rasgo más significativo del diseño in-situ es que permite potenciar la percepción del equipo multidisciplinario, el cual podría apreciar además el lugar desde la perspectiva del alumno.

El contenido educativo puede involucrar características puntuales del ambiente o no, esto es lo que se denomina en la Fig. 2 como contenidos educativos contextuales o generales respectivamente. En el caso de diseñar contenidos educativos contextuales su reuso se verá acotado a lugares con iguales características, por ejemplo, dos jardines botánicos con el mismo ambiente (por ejemplo, un contenido educativo que involucra observar un especie particular de árbol, requiere que la misma especie esté presente en ambos jardines botánicos). Esto determina el reuso de los contenidos educativos, siempre y cuando éstos sean generales o en el caso de ser contextuales su reuso se limita a espacios con similares características.

No hay que perder de vista, que al diseñar contenidos educativos (ya sean contextuales o no) se deben considerar tanto los aspectos educativos (por ejemplo, objetivo del contenido, temática, outcome y rango de edades de los alumnos destinatarios) como ciertos aspectos tecnológicos relacionados al uso de los dispositivos móviles (por ejemplo, el tamaño de pantalla podría limitar la cantidad de información definida para el contenido educativo). En [32] se presenta un análisis del efecto en la percepción del usuario referido al uso de dispositivos móviles inteligentes con pantallas grandes.

\section{Estructurar los Contenidos Educativos}

Esta etapa se debe realizar luego de diseñar los contenidos educativos. La organización de estos contenidos en educación tiene sus bases ya establecidas [33]. En el caso de Aplicaciones Educativas Móviles basadas en Posicionamiento, hay que tener en cuenta que, luego estas estructuras definirán la forma en la que los alumnos se mueven dentro del espacio físico. Por ejemplo, una estructura lineal hará que los alumnos se muevan secuencialmente de un contenido educativo a otro. Este tema se puede explorar, por ejemplo, en [34] donde se plantean diferentes metáforas para guías móviles. A su vez, [34] fue usada como base por [35] para la conceptualización de juegos educativos móviles basados en posicionamiento y por [21] para estructurar narrativas hipermediales basadas en posicionamiento.

Esta estructuración del contenido educativo se debe definir independiente de los lugares relevantes en los que posteriormente se podría brindar dichos contenidos, al estilo de lo que se menciona en [35].

Cada una de las estructuras está conformada por elementos. Para cada elemento se debe indicar que contenido educativo se utiliza. Como se representó en la Fig. 1.

Dado que estas estructuras funcionan de manera igual, sin importar el contenido educativo que contienen, estas podrían ser pensadas como Plantillas (o templates), definiendo de esta 
manera un funcionamiento general. Por ejemplo, una estructura lineal podría siempre funcionar de la misma manera. Esto se podría diseñar usando el patrón de diseño Template [36] o bien considerar que son distintas estrategias para recorrer elementos, y en ese caso, usar el patrón de diseño Strategy [36].

\section{Relacionar los Elementos de las estructuras con los Lugares Relevantes}

Teniendo los lugares relevantes identificados y habiendo definido la estructuración de los contenidos educativos, se puede proseguir a relacionar los elementos de las estructuras con los lugares relevantes. Esta relación, es la que determina el "weaving" entre los concerns de estructuras y de posiciones. Siempre que se separa en concerns debe de definirse un punto de unión entre los mismos.

Podría suceder que en un lugar relevante se brinde más de un elemento de una estructura. También podría suceder que diferentes elementos de estructuras sean brindados en un mismo lugar relevante, como se presentó en la Fig. 1. Este reuso es posible cuando los lugares relevantes y los elementos de las estructuras son definidos de manera independiente uno del otro.

\section{DISCUSIÓN}

En esta sección se discuten diferentes características a tener en cuenta en la co-creación de Aplicaciones Educativas Móviles basadas en Posicionamiento. Además, se detallan distintos trabajos relacionados con el framework propuesto, mencionando diferencias y similitudes.

En la Sección 3 se pudo apreciar que hay aspectos que se pueden reusar, dependiendo de cómo los mismos fueron codiseñados. Las estructuras definidas con contenidos educativos generales se podrán reusar en cualquier lugar físico. Mientras que en aquellas estructuras definidas con contenidos educativos contextuales su reuso estará limitado a lugares físicos con características similares. En el caso de los lugares relevantes, éstos pueden combinarse con cualquier estructura.

El framework propuesto está definido para ser usado por un equipo multidisciplinario. Además, los expertos en tecnología podrían usar un enfoque de modelado como el propuesto en [22], el cual permite considerar aspectos específicos de este tipo de aplicaciones desde la perspectiva de la separación de concerns. Un prototipo específico es presentado en [22], el cual puede ser usado como base de cómo se pueden diseñar e implementar este tipo de aplicaciones.

Para el planteo del framework conceptual se utilizó lo aprendido respecto al modelado en la ingeniería de software. En particular, se han explorado diferentes temáticas, por ejemplo, modelos de variabilidad en aplicaciones sensibles al contexto [30], separación de concerns en hipermedia móvil [37], modelado de actividades educativas móviles basadas en posicionamiento [22] y diseño de una herramienta de autor insitu para aplicaciones sensibles a la posición [24]. Esto ha contribuido junto con la bibliografía existente al planteo del framework propuesto. En particular, buscando el reuso de los aspectos involucrados.
Usando el framework propuesto se pueden co-diseñar Aplicaciones Educativas Móviles basadas en Posicionamiento. La siguiente etapa (como se mencionó en la Sección 3) es realizar el ciclo de desarrollo usando tecnología móvil y tomando como base el co-diseño conceptual realizado. Este ciclo debe seguir las buenas prácticas de la ingeniería de software, por ejemplo, la separación de concerns (a fin de mantener el reuso de cada uno de los aspectos co-diseñados) y el uso de patrones de diseño [36], los cuales permiten flexibilidad a la hora extender o modificar los desarrollos. Por ejemplo, se podía usar el patrón de diseño Observer [36], para monitorear los cambios de la posición del alumno. Esto permitiría, entre otras cosas, desacoplar en al menos un nivel los mecanismos de sensado, ya que se da aviso que el alumno cambio la posición, pero no es relevante conocer que mecanismo lo sensó. Además, esto da la flexibilidad de que si se cambia $o$ se agregan nuevos mecanismos de posicionamiento el resto de la aplicación no se ve afectada. Esta etapa será abordada en un futuro, para poder brindar lineamientos que ayuden al equipo multidisciplinario en este ciclo.

En el caso de no seguir buenas prácticas, el desarrollo quedará acoplado, por ejemplo, el contenido educativo con los lugares relevantes. Es decir, se obtienen desarrollos ad-hoc donde el reuso no es posible, como por ejemplo, sucede en [38], [39] y [40].

Existen actualmente diversos frameworks conceptuales para aprendizaje móvil que contemplan la etapa de diseño como parte de los mismos. Por ejemplo, en [10] el framework conceptual consta de seis etapas, una de ellas contempla el diseño de los contenidos. Sin embargo, al estar el framework orientado a aplicaciones educativas móviles en general, no se aborda el posicionamiento de los contenidos educativos en la etapa de diseño. En particular, para el aprendizaje móvil basado en posicionamiento, en [2] se presenta un framework conceptual focalizado en contemplar el contexto en el aprendizaje móvil y tomar datos in-situ. Este framework se compone de tres componentes: facilitación, actividad in-situ y tecnología para el diseño de aprendizaje móvil contextual. En [2] se presenta un ejemplo específico del uso del framework, donde en base al mismo se detalla el diseño de una actividad y su estructuración. Para la realización del mismo los docentes recorren in-situ el lugar. Sin embargo, como parte del framework no se presentan lineamientos para representar el lugar físico donde se usará la aplicación (es decir, lo que se menciona como bosquejo), así como tampoco lineamientos de diseño para fomentar el reuso de la actividades ni de los lugares relevantes que identifican para brindar sus actividades. Otro framework conceptual es presentado en [41], el cual está orientado a actividades educativas mediadas por tecnología móvil. Dicho framework consta de cinco fases, las cuales se presentan mediante un caso concreto de aplicación. En particular, cuando diseñan, identifican los elementos de interés in-situ, por ejemplo, árboles y plantas a los cuales les colocan códigos QR para que luego, los alumnos usando la aplicación móvil in-situ, puedan escanearlos y obtener información relevante a la especie en cuestión. En el framework los autores 
identifican dos perspectivas: la relacionada a lo educativo (por ejemplo, para la definición y secuenciación de tareas educativas) y la inherente a las ciencias de la computación (que contempla, por ejemplo, aspectos tecnológicos y de implementación). En [41] si bien se contempla la identificación in-situ de elementos, no se propone el reuso de lugares relevantes, ni de tareas o actividades educativas. Acorde a lo antes mencionado, se puede apreciar que existen diferentes trabajos en donde se considera el diseño in-situ, sin embargo estos trabajos ([10], [2] y [41]) no se focalizan en el reuso de los aspectos diseñados como se presenta en el framework propuesto.

Como se pudo apreciar en la Sección 3 hay otras etapas además del diseño conceptual que se deben explorar en relación a la temática de la co-creación de Aplicaciones Educativas Móviles basadas en Posicionamiento. Se desea que este trabajo sirva para fomentar la discusión en esta temática, respecto del reuso y las posibles buenas prácticas a emplear en las etapas identificadas.

El framework conceptual está en una etapa inicial, a futuro se pondrá en práctica con un equipo multidisciplinario para poder validar la viabilidad y usabilidad del mismo, y con estos resultados realizar los ajustes de especificidad necesarios para que este sea útil en el co-diseño de Aplicaciones Educativas Móviles basadas en Posicionamiento. Usar un framework para el co-diseño no es una tarea trivial, sino que requiere de un entrenamiento específico para el equipo multidisciplinario ([2] y [14]).

\section{CONCLUSIONES Y TRABAJOS FUTUROS}

En este trabajo se presentó un Framework Conceptual para el co-diseño de Aplicaciones Educativas Móviles basadas en Posicionamiento por medio de un equipo multidisciplinario. En relación con este tipo de aplicaciones se identifican cuatro etapas relacionadas, como son: diseño conceptual, ciclo de desarrollo usando tecnología móvil, puesta en práctica del desarrollo realizado y evaluación de la puesta en práctica. En particular, el framework propuesto aborda la primera etapa, el diseño conceptual.

El framework propuesto brinda los lineamientos para diseñar el contenido educativo desacoplado de los lugares relevantes identificados dentro de un espacio físico (ya sea indoor como outdoor). Esto facilita el reuso de ambos conceptos en diferentes aplicaciones.

Se identificaron cuatro concerns como parte del framework: diseño in-situ del espacio físico, diseño de los contenidos educativos, estructuración de los contenidos educativos y relaciones entre los elementos de las estructuras con los lugares relevantes. Dependiendo de cómo se plantean cada uno de estos concerns, será la factibilidad de reuso de los mismos, como se mencionó en Sección 3. Por ejemplo, las estructuras definidas con contenidos educativos generales se podrían reusar en cualquier lugar físico o los lugares relevantes identificados podrían combinarse con cualquier elemento de una estructura.

Como trabajo futuro, a partir del framework conceptual propuesto se planificarán encuentros de entrenamiento con equipos multidisciplinarios, para poder determinar la utilidad del mismo, y así relevar cualquier característica emergente que pueda contribuir a enriquecer el framework.

Además, se trabajará en el abordaje de las otras etapas identificadas en este trabajo, como son: ciclo de desarrollo usando tecnología móvil, puesta en práctica del desarrollo realizado y evaluación de la puesta en práctica. Buscando establecer para cada una de éstas lineamientos que brinden soporte significativo al equipo multidisciplinario.

\section{REFERENCIAS}

[1] C. Emmanouilidis, R.A. Koutsiamanis, and A. Tasidou, "Mobile guides: Taxonomy of architectures, context awareness, technologies and applications," Journal of Network and Computer Applications, vol. 36, no. 1, Jan. 2013, pp. 103-125, doi:10.1016/j.jnca.2012.04.007.

[2] E. Tan and H. J. So, "Rethinking the impact of activity design on a mobile learning trail: The missing dimension of the physical affordances," IEEE Transactions on Learning Technologies, vol. 8, no. 1, Mar. 2015, pp. 98-110, doi: 10.1109/TLT.2014.2376951.

[3] S. McKenney, "Designing and researching technology-enhanced learning for the zone of proximal implementation," Research in learning technology, vol. 21, Sep. 2013, doi:10.3402/rlt.v21i0.17374.

[4] Y. Kali, S. McKenney, and O. Sagy, "Teachers as designers of technology enhanced learning," Instructional science, vol. 43, no. 2, Mar. 2015, pp. 173-179, doi:10.1007/s11251-014-9343-4.

[5] S. McKenney, Y. Kali, L. Markauskaite, and J. Voogt, "Teacher design knowledge for technology enhanced learning: an ecological framework for investigating assets and needs," Instructional Science, vol. 43, no. 2, Mar. 2015, pp. 181-202, doi:10.1007/s11251-014-9337-2.

[6] C. O'Malley, G. Vavoula, J. Glew, J. Taylor, M. Sharples, P. Lafrere, P. Lonsdane, L. Naismith and J. Waycott, "Guidelines for learning/teaching/tutoring in a mobile environment," Public deliverable from the MOBILearn project (D.4.1), Mar. 2005, https://hal.archives-ouvertes.fr/hal-00696244/document.

[7] J. Traxler, "Learning in a mobile age," International Journal of Mobile and Blended Learning (IJMBL), vol. 1, no. 1, Jan. 2009, pp. 1-12, doi:10.4018/jmbl.2009010101.

[8] U. Lucke, and C. Rensing, "A survey on pervasive education," Pervasive and Mobile Computing, vol.14, no. C, Oct. 2014, pp. 3-16, doi: 10.1016/j.pmcj.2013.12.001.

[9] P. Harpur and M.R. De Villiers, "MUUX-E, a framework of criteria for evaluating the usability, user experience and educational features of m-learning environments," South African Computer Journal, vol. 56, no. 1, Jul. 2015, pp. 1-21, doi: 10.18489/sacj.v56i1.240.

[10] O.R.B. Figueredo and J.A.J. Villamizar "Framework for Design of Mobile Learning Strategies," in Handbook of Mobile Teaching and Learning, Springer Berlin Heidelberg, Jan. 2015, pp. 75-89, doi: 10.1007/978-3-642-54146-9_87.

[11] A. Peña-Ayala and L. Cárdenas "A Revision of the Literature Concerned with Mobile, Ubiquitous, and Pervasive Learning: A Survey," in Mobile, Ubiquitous, and Pervasive Learning, Springer International Publishing, vol. 406, Dec. 2015, pp. 55100, doi: 10.1007/978-3-319-26518-6_3.

[12] R. Cober, E. Tan, J. Slotta, H.J. So, and K.D. Könings, "Teachers as participatory designers: Two case studies with 
technology-enhanced learning environments," Instructional Science, vol. 43, no. 2, Mar. 2015, pp. 203-228, doi: 10.1007/s11251-014-9339-0.

[13] C. L. Lai and G. J. Hwang, "High school teachers' perspectives on applying different mobile learning strategies to science courses: the national mobile learning program in Taiwan," International Journal of Mobile Learning and Organisation, vol. 9, no. 2, Jul. 2015, pp. 124-145, doi: 10.1504/IJMLO.2015.070704.

[14] D. Parsons, J. Wishart and H. Thomas, "Exploring Mobile Affordances in the Digital Classroom," Proc. 12th International Conference on Mobile Learning (ML 2016), Curran Associates, Jan. 2016, pp. 43-50, http://davidparsons.ac.nz/papers/Exploring\%20Mobile\%20Affor dances.pdf.

[15] M. Katsamani and S. Retalis, "Orchestrating learning activities using the CADMOS learning design tool," Research in Learning Technology, vol. 21, no. 1, Sep. 2013, doi: 10.3402/rlt.v21i0.18051.

[16] P. Tarr, H. Ossher, W. Harrison, and S.M. Sutton, "N Degrees of Separation Multi-Dimensional Separation of Concerns," Proc. 21st international conference on Software engineering (ICSE '99), ACM Press, May. 1999, pp. 107-119, doi: $10.1145 / 302405.302457$

[17] D. Weyns, M. Caporuscio, B. Vogel, and A. Kurti, "Design for Sustainability $=$ Runtime Adaptation UEvolution," Proc. 2015 European Conference on Software Architecture Workshops (ECSAW '15), ACM Press, Sep. 2015, pp. 62-69, doi: $10.1145 / 2797433.2797497$

[18] Jardín Botánico "Carlos Thays", https://turismo.buenosaires.gob.ar/es/atractivo/jard\%C3\%ADnbot\%C3\%A1nico. Fecha de último acceso: 2017-05-08.

[19] H. Löwen, A. Schwering, J. Krukar and S. Winter, "Perspectives in Externalizations of Mental Spatial Representations," in International Conference on Geographic Information Science (AGILE 2017), Springer, May. 2017, pp. 111-127, doi: 10.1007/978-3-319-56759-4_7 .

[20] S. Zhou, S. Winter, M. Vasardani and S. Zhou, "Place descriptions by landmarks," Journal of Spatial Science, vol. 62, no. 1, Mar. 2017, pp. 47-67, doi: 10.1080/14498596.2016.1196623.

[21] D.E. Millard and C. Hargood, "Location Location Location: experiences of authoring an interactive location-based narrative," Proc. 9th International Conference on Interactive Digital Storytelling (ICIDS 2016), Springer, Nov. 2016, pp. 419-422, doi:10.1007/978-3-319-48279-8 40.

[22] A.B. Lliteras, "Un enfoque de modelado de actividades educativas posicionadas que contemplan elementos concretos," Master Thesis, Faculty of Informatics, National University of La Plata, Argentina, Nov. 2015, http://hdl.handle.net/10915/50030.

[23] A.M. Zimbello, F.M. Alconada Verzini, C. Challiol, A.B. Lliteras and S.E. Gordillo, "Authoring Tool for Location-based Learning Experiences," Proc. 4th IEEE/ACM International Conference on Mobile Software Engineering and Systems, IEEE Press, May. 2017, pp. 211-212, doi: 10.1109/MOBILESoft.2017.32.

[24] F.M. Alconada Verzini, J.I. Tonelli, C. Challiol, A.B. Lliteras, and S.E. Gordillo, "Authoring Tool for Location-Aware Experiences," Proc. 2015 Workshop on Narrative \& Hypertext, ACM Press, Sep. 2015, pp. 21-25, doi: 10.1145/2804565.2804570.
[25] H. Kato, D. Chai and K.T. Tan, "Barcodes for mobile devices," Cambridge: Cambridge University Press, Jul. 2010, doi: 10.1017/CBO9780511712241

[26] GPS, http://www.gps.gov/systems/gps/. Fecha de último acceso: 2017-05-08.

[27] XML, https://www.w3.org/standards/techs/xml\#w3c_all. Fecha de último acceso: 2017-05-08.

[28] J. Wang and M. Worboys, "Ontologies and representation spaces for sketch map interpretation," International Journal of Geographical Information Science, vol. 31, no. 9, Apr. 2017, pp. 1697-1721, doi: 10.1080/13658816.2017.1309043.

[29] S. Jan, A. Schwering, M. Chipofya and T. Binor, "Qualitative representations of extended spatial objects in sketch maps," in Connecting a Digital Europe Through Location and Place, Springer International Publishing, May. 2014, pp. 37-54, doi: 10.1007/978-3-319-03611-3_3.

[30] A. Fortier, G. Rossi, S.E. Gordillo and C. Challiol, "Dealing with variability in context-aware mobile software," Journal of Systems and Software, vol. 83, no. 6, Jun. 2010, pp. 915-936, doi: 10.1016/j.jss.2009.11.002.

[31] P. Santos, D. Hernández-Leo, and J. Blat, "To be or not to be in situ outdoors, and other implications for design and implementation, in geolocated mobile learning," Pervasive and Mobile Computing, vol. 14, Oct. 2014, pp. 17-30, doi: 10.1016/j.pmcj.2013.09.001

[32] K. J. Kim and S.S. Sundar, "Mobile persuasion: Can screen size and presentation mode make a difference to trust?," Human Communication Research, vol. 42, no. 1, Jan. 2016, pp.45-70, doi: 10.1111/hcre.12064

[33] Á. Díaz-Barriga, "TIC en el trabajo del aula: Impacto en la planeación didáctica," Revista Iberoamericana de Educación Superior (RIES), México, UNAM-IISUE/Universia, vol. 4, no. 10, May. 2013, pp. 3-21, doi: 10.1016/S2007-2872(13)71921-8.

[34] J. Kjeldskov and J. Paay, "Augmenting the City with fiction: fictional requirements for mobile guides," Mobile Interaction with the Real World (Mobile HCI'07), Springer-Verlag Berlin, vol. 5, Sep. 2007, pp. 41-55, http://www.medien.ifi.lmu.de/mirw2007/papers/MGuides2007_ Kjeldskov.pdf

[35] A.B. Lliteras, C. Challiol and S.E. Gordillo, "Juegos educativos móviles: aspectos involucrados," in XVIII Congreso Argentino de Ciencias de la Computación, Oct. 2013, pp. 671-680, http://hdl.handle.net/10915/32304.

[36] E. Gamma, R. Helm, R. Johnson, and J. Vlissides, "Design patterns: elements of reusable object-oriented software," Addison-Wesley Longman Publishing Co, 1995.

[37] C. Challiol, G. Rossi, S.E. Gordillo and A. Fortier, "Separation of concerns in mobile hypermedia: architectural and modeling issues," Handbook of Research on Mobile Software Engineering: Design, Implementation and Emergent Applications, vol. 1, May. 2012, pp. 211-233, doi: 10.4018/9781-61520-655-1.

[38] P. Santos, M. Pérez-Sanagustín, D. Hernández-Leo and J. Blat, "QuesTInSitu: From tests to routes for assessment in situ activities," Computers \& Education, vol. 57, no. 4, Dec. 2011, pp. 2517-2534, doi: 10.1016/j.compedu.2011.06.020.

[39] S. Gómez, P. Zervas, D. G. Sampson and R. Fabregat, "Contextaware adaptive and personalized mobile learning delivery supported by UoLmP," Journal of King Saud UniversityComputer and Information Sciences, vol. 26, no. 1, Jan. 2014, pp. 47-61, doi: 10.1016/j.jksuci.2013.10.008. 
[40] T. C. Liu, Y. C. Lin and F. Paas, "Effects of prior knowledge on learning from different compositions of representations in a mobile learning environment," Computers \& Education, vol. 72, Mar. 2014, pp. 328-338, doi: 10.1016/j.compedu.2013.10.019.
[41] J. Nouri, D. Spikol and T.A. Cerratto-Pargman, "A Learning Activity Design Framework for Supporting Mobile Learning," Designs for Learning, vol. 8, no. 1, Jan. 2016, pp. 1-12, doi: 10.16993/dfl.67. 